\title{
Discovery of Potent and Selective p53-MDM2 Protein-Protein Interaction Inhibitors as Anticancer Drugs
}

\author{
Philipp Holzer
}

\begin{abstract}
As a result of our persistent efforts to discover novel inhibitors of the p53-MDM2 protein-protein interaction useful for the treatment of cancer, the potent and selective MDM2 inhibitors NVP-CGM097 and NVP-HDM201 with excellent in vitro and in vivo profile were selected as clinical candidates and are currently in phase 1 clinical development. This short review article provides a summary of the program history, the applied pharmacophore model and the discovery story of these novel p53-MDM2 inhibitor investigational drugs.
\end{abstract}

Keywords: Clinical trial · HDM2 · Inhibitor · MDM2 · NVP-CGM097 · NVP-HDM201 · p53 · Protein-protein interaction · PPI

The p53 tumor suppressor protein is a transcription factor that regulates cellular responses to DNA damage and stress stimuli by inducing cell-cycle arrest, DNA repair, apoptosis, or senescence. ${ }^{[1]}$ With this essential role, the $\mathrm{p} 53$ protein has often been regarded as the 'guardian of the human genome'. [2] Upon cellular stress, p53 can arrest cell cycle progression, allowing the DNA to be repaired or it may lead to apoptosis. These functions are achieved by the trans-activational properties of $\mathrm{p} 53$, which activate a series of genes involved in cell cycle regulation. In tumor cells, p53 is frequently inactivated, often via over-expression of MDM2 (mouse double minute 2 homolog, also known as HDM2, the human homolog), a protein which has ubiquitin ligase activity and binds p53 with high affinity, thereby promoting its degradation as well as masking its ability to activate transcription. ${ }^{[3]}$ While mice lacking p53 develop normally, they are disposed to the development of a variety of tumors. ${ }^{[4]} \mathrm{It}$ has been estimated that p53 mutations are the most frequent genetic events in human cancers and account for more than $50 \%$ of all cases. ${ }^{[5]}$ Even though TP53, the gene

${ }^{*}$ Correspondence: Dr. Ph. Holzer Novartis Institutes for BioMedical Research $\mathrm{CH}-4002$ Basel

E-mail: Philipp.Holzer@novartis.com encoding the p53 protein, retains wildtype status in the remaining $50 \%$ of human cancers, the regulation and activation of p53 consist of a complex mechanism and p53 stabilization involves multiple layers of MDM2 regulation. ${ }^{[6]}$ Whereas p53 activation underlies the activity of many DNA-damaging chemotherapeutic agents in response to cellular stresses, a more attractive therapeutic strategy would be to competitively bind MDM2 and restore p53 levels to a threshold that induces apoptosis. ${ }^{[7]}$ As a consequence, substantial drug discovery efforts have been directed over the last twenty years using numerous strategies to block the protein-protein interaction between p53 and MDM2.

Protein-protein interactions (PPIs) often show poorly drugable shallow protein interfaces that span relatively large surface areas $\left(>1600 \AA^{2}\right)$ and typically lack the deep, well-defined binding pockets which are attractive for small molecule drug discovery. ${ }^{[8]}$ Kussie and coworkers published the first X-ray crystal structure of the transactivation domain of p53 in complex with the binding domain of MDM2 and this showed that the interaction relied upon a 'hot-spot' of a triad of p53 amino acids. [9] The side-chains of Phe-19, Trp-23 and Leu-26 in positions $\mathrm{i}, \mathrm{i}+4$ and $\mathrm{i}+7$ along one helical face of p53 have extended conformations and pack closely to each other, leading to an array of van der Waal's contacts and formation of two H-bonds with the surface of MDM2. Complementary work published by Novartis scientists showed that an octamer peptide incorporating the same three residues, but with a 6-chlorotryptophan replacement, potently inhibited ( $\mathrm{IC}_{50}$ of $5 \mathrm{nM}$, compound 1) the p53-MDM2 interaction. ${ }^{10]}$ Even though the initial interest in polypeptides to tar- get the p53/MDM2 interaction was limited due to their poor membrane permeability and physiological instability, these early studies provided a pharmacophore model that could be exploited with drug-like molecules for this target.

Using our acquired knowledge of key pharmacophore elements, in silico approaches were used to support the identification for novel hits for this target. In this context, the 'central valine concept' was created using molecular modeling to orient in 3D the pharmacophore elements and connect them to a central core. ${ }^{[11]}$ Placing a planar aromatic or hetero-aromatic core moiety within van der Waals distance of Val-93, a residue occupying a central position in the p53 binding pocket of MDM2, provided a core platform that offered appropriate vectors to occupy the three essential sub-pockets of the MDM2 cleft involved in the protein-protein interaction. This led to the discovery of two new p53-MDM2 inhibitor scaffolds, one based on a 3-imidazolyl substituted indole[11] (compound 2) and the other on a tetra-substituted imidazole core structure ${ }^{[12]}$ (compound 3). Representative lead structures are shown in Fig. 1. Structure-guided derivatization of the initial hits provided compounds with low nanomolar affinity to MDM2 in a biochemical TR-FRET assay, which had cellular potencies in the same range as that of the well-established prototype p53-MDM2 inhibitor Nutlin-3a $\left(\mathrm{EC}_{50}=1.9 \mu \mathrm{M}\right)$.

Parallel to these structure-based efforts, we sought to extend chemical space by large scale knowledge-based virtual screening for new p53-MDM2 inhibitor chemotypes. ${ }^{[13]}$ Following a procedure previously published, ${ }^{[14]}$ which involved a combination of $2 \mathrm{D}$ and $3 \mathrm{D}$ virtual screening methods, around 50'000 compounds 
Fig. 1. Chemical structures of Novartis' early hit compounds that bind to MDM2. Compound 1, containing several nonnatural amino acids, was the first potent octapeptide binding to MDM2 and it formed the basis for the design of nonpeptidic compounds. Developing and applying the 'central valine concept' allowed the identification of the imidazolyl substituted indole series as represented by compound 2 and the tetrasubstituted imidazole series as represented by 3 .

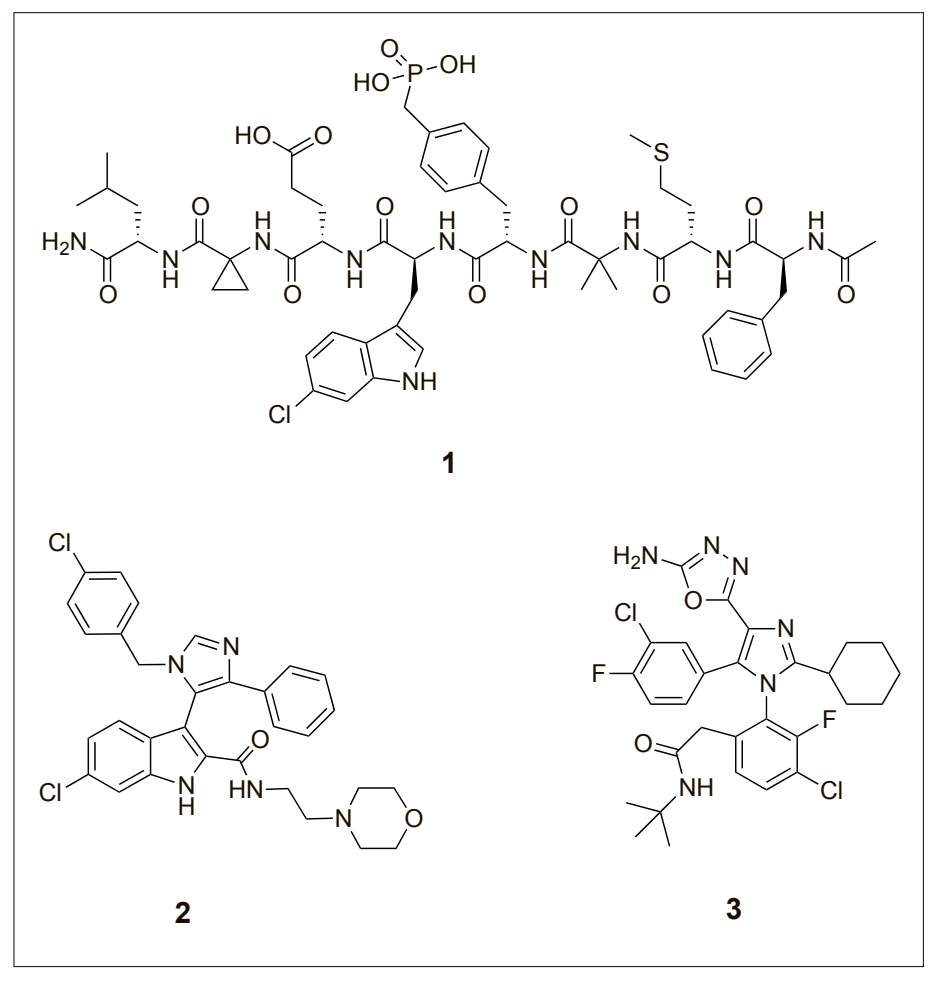

from the Novartis compound collection were selected to be tested in our p53MDM2 program. From this effort, the dihydroisoquinolinone derivative 4 was identified as an interesting hit compound that inhibited the p53-MDM2 interaction with an $\mathrm{IC}_{50}$ value of $0.54 \mu \mathrm{M}$ in the TR-FRET assay, which translated into weak antiproliferative activity in cells. Compound $\mathbf{4}$ was considered to be an attractive starting point for further exploration. To guide the design of analogs, a binding mode was hypothesized for compound $\mathbf{4}$ based on the pharmacophore knowledge from the established chemical classes (Fig. 2). In this model, which followed the "central valine concept', the dihydroisoquinolinone core of 4 is in van der Waals contact to Val-93. Such a position of the bicyclic ring system in the cavity allows the ethoxy groups and the chloro-phenyl ring to occupy the Phe-19 and the Trp-23 sub-pockets, respectively, while the methoxy-phenyl ring forms a $\pi-\pi$ stacking interaction with His96 in the Leu-26 sub-pocket. In addition, the carbonyl group of compound $\mathbf{4}$ can accept a hydrogen bond from the imidazole ring of His-96 as previously seen within of our imidazole series. ${ }^{[12]}$ The chlorophenyl moiety sitting in the 'hot-spot' Trp-23 sub-pocket is consistent with the binding mode of previous p53-MDM2 binders and goes back to the initial peptide work and was consistent with the available crystallographic pharmacophore understanding.

Optimization of compound $\mathbf{4}$ started with the variation of the methoxy group on the phenyl ring occupying the Leu-26 subpocket. Based on the binding model and the structure-activity relationships (SAR) of our previous series of p53-MDM2 inhibitors, chloro-substituents were expected to improve potency. ${ }^{[14]}$ However, this initial SAR work was difficult to interpret and we focused on obtaining a cocrystal structure of MDM2 in complex with a representative derivative of the dihydroisoquinolinones. First attempts were unsuccessful due to the low solubility of the compounds and consequently solubilizing groups were added to the scaffold to allow adequate concentrations of the ligand to be achieved in co-crystallization experiments. As described previously, ${ }^{[14]}$ this exercise successfully resulted in the first co-crystal structure of MDM2 bound to the dihydroisoquinolinone derivative $\mathbf{5}$ (Fig. 3). To our surprise, this revealed an unprecedented binding mode in which the bicyclic core of the inhibitor made hydrophobic contact with residues Iso-54, Phe-55 and Glu-58 of MDM2 rather than interacting with Val-93 as proposed in the PHE (Phe-19), TRP (Trp-23) and LEU (Leu-26). initial binding model. This novel binding mode appears to be facilitated by the sidechain of Phe-55, which through a rotation allows the formation of an intramolecular face-to-edge aryl-aryl interaction. Besides these new interactions, the compound was located in the center of the MDM2 cavity where it occupied the three sub-pockets that contribute to the overall binding affinity of the inhibitor. The chlorophenyl ring occupied the usual 'hot-spot' Trp-23 sub-pocket, but the other sub-pockets were occupied differently. Through a rotation of the molecule in the MDM2 binding cavity, compound 5 did not form a $\pi-\pi$ stacking interaction with His-96 within the Leu-26 sub-pocket, but occupied this cavity with its two ethoxy substituents with the substituted phenyl ring pointing towards the Phe19 sub-pocket. This new way of interacting with the p53-MDM2 binding site formed the basis for the further optimization of our dihydroisoquinolinone series.

The co-crystal structure of compound 5 suggested that expanding the region of the compound occupying the Phe-19 subpocket could improve binding interactions with MDM2. On this basis rapid progress resulted in a new lead compound $\mathbf{6}$. After separation of the two diastereoisomers, the $S$-configuration of compound 6 represented the first member of this new series that achieved single digit nanomolar activity in the biochemical assay, as well as potency in the cellular proliferation assay similar to that of the reference p53-MDM2 inhibitor Nutlin-3a. Further medicinal chemistry exploration and extensive optimization culminated in the discovery of NVPCGM097, the first Novartis p53-MDM2 inhibitor compound to successfully progress through toxicology studies and into phase 1 clinical trials, which has been described in detail in refs. [15] and [16].

The binding mode of NVP-CGM097 is exemplified in Fig. 4. The dihydroisoquinolinone core of NVP-CGM097 sits in the center of the binding site and arranges for the appropriate exit vectors to extend into the three critical binding pockets of

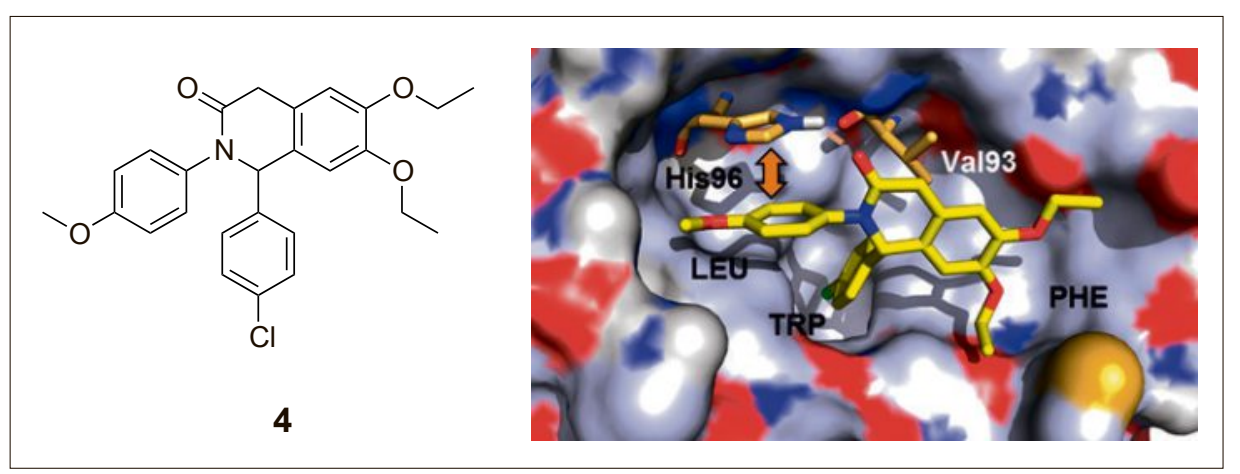

Fig. 2. Compound 4 identified from a 50'000 compound screen based on a 2D and 3D virtual screen in the initial binding model in the MDM2 cavity. The three MDM2 sub-pockets are labeled 
<smiles>CCOc1cc2c(cc1OCC)C(c1ccc(Cl)cc1)N(c1ccc(C)cc1OCc1nnn[nH]1)C(=O)C2</smiles>

5<smiles>CCOc1cc2c(cc1OC(C)CC)C(=O)N(c1ccc(N(C)Cc3ccncc3)cc1)C2=O</smiles>

6

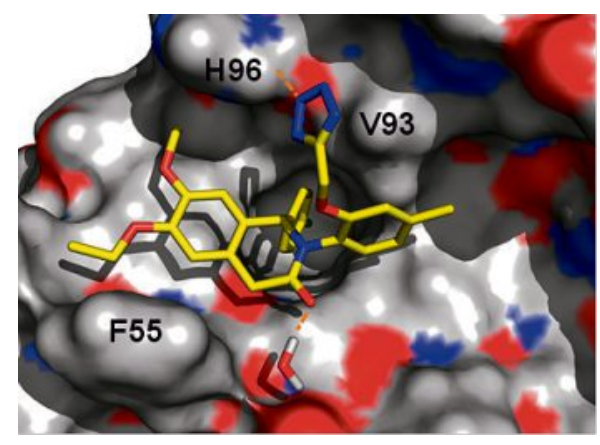

Fig. 3. X-ray co-crystal structure of MDM2 in complex with compound 5 (PDB code: 4ZYC). The tetrazole forms a hydrogen bond with His-96 and through a rotation of the compound. The three sub-pockets are occupied by different groups of the molecule as originally anticipated. Supported by knowledge gained from this X-ray co-crystal structure, structure-based design guided the optimization and lead compound $\mathbf{6}$ has been identified reaching nanomolar potency in the biochemical assay. The $S$-configuration of the compound 6 binds to the MDM2 pocket.

the p53 residues Leu-26, Trp-23, and Phe19. The isopropyl and the methyl ethers fill the Leu-26 sub-pocket with the ether oxygens participating in water-mediated H-bond interactions. The 4-chlorophenyl group reaches deep into the Trp-23 binding cavity. The front of the pocket is closed as Phe-55 swings up and displays a faceto-edge interaction with the dihydroisoquinolinone scaffold. As earlier described, the movement of Phe-55 is specific to this scaffold. ${ }^{[15]}$ The carbonyl function of the dihydroisoquinolinone core is engaged in a water-mediated hydrogen bond interaction with the carbonyl oxygen of Phe-55 and therefore contributes to the overall affinity of the molecule. But more notably, it induces a conformational constraint to the proximal $\mathrm{N}$-aryl side-chain and as a consequence, the right torsion angle is provided to efficiently enter the Phe-19 binding region. The dialkylated aniline functionality affords the proper exit vector and projects the side chain to nicely occupy the central part of the Phe-19 binding region. Finally, the N-methyl piperazinone motif binds towards the 'mouth' of the Phe-19 binding cavity in a water-rich region to nestle perfectly between the protein walls, contributing significantly to the potency of NVP-CGM097.[15]

The in vivo anti-tumor activity of NVPCGM097 was assessed in a p53 sensitive and MDM2-amplified SJSA-1 human tumor xenograft in rats. The compound induced $>85 \%$ tumor regression when dosed once daily at $30 \mathrm{mg} / \mathrm{kg}$ (Fig. 5). Intermittent three times per week treatment ( $3 \mathrm{qw}$, treatment on Monday, Wednesday, and Friday) dose-dependently and substantially slowed SJSA-1 tumor growth inducing a T/C of $25 \%$ at a dose of $30 \mathrm{mg} / \mathrm{kg}$ and resulting in full tumor regression when dosed at 70 $\mathrm{mg} / \mathrm{kg} \cdot{ }^{[15]}$

While NVP-CGM097 progressed into clinical phase 1 trials in cancer patients bearing p53 wild-type tumors, the chemistry team revisited the initial binding con-

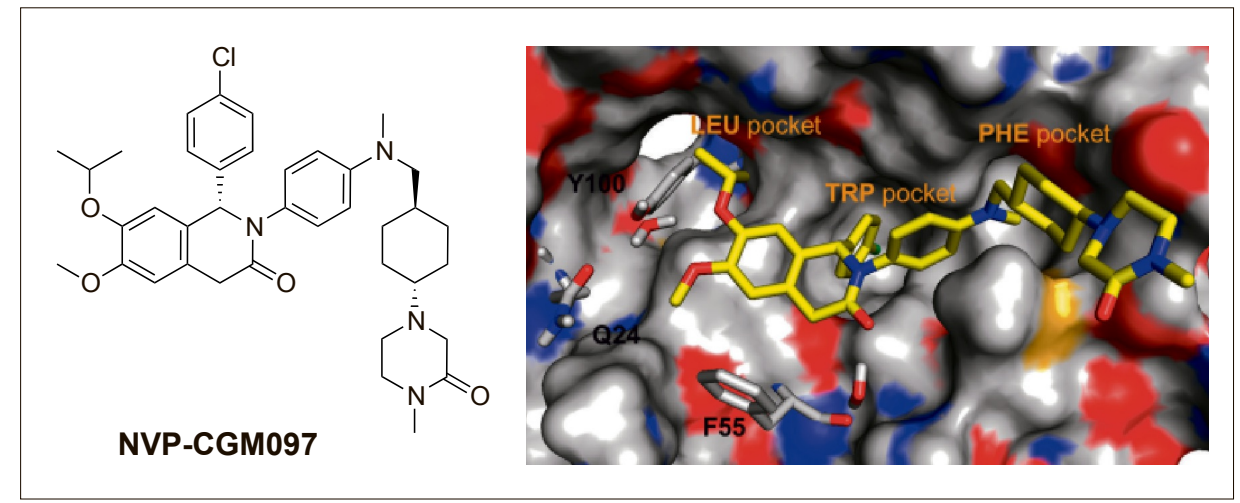

Fig. 4. X-ray co-crystal structure of NVP-CGM097 bound to human MDM2 at $1.80 \AA \AA$ resolution (PDB code: 4ZYF). MDM2 binding pockets are labeled in yellow font based on the p53 side chains which interact in these regions.

Fig. 5. In vivo antitumor activity of NVP-CGM097. Tumor bearing rats (subcutaneous SJSA-1 xenografts) received either vehicle daily (q24h, black), $30 \mathrm{mg} /$ $\mathrm{kg}$ daily (orange), 30 $\mathrm{mg} / \mathrm{kg}$ three times a week (3qw, yellow), or $70 \mathrm{mg} / \mathrm{kg}$ three times a week (blue) administered as an oral suspension of NVP-CGM097 in $0.5 \%$ HPMC.

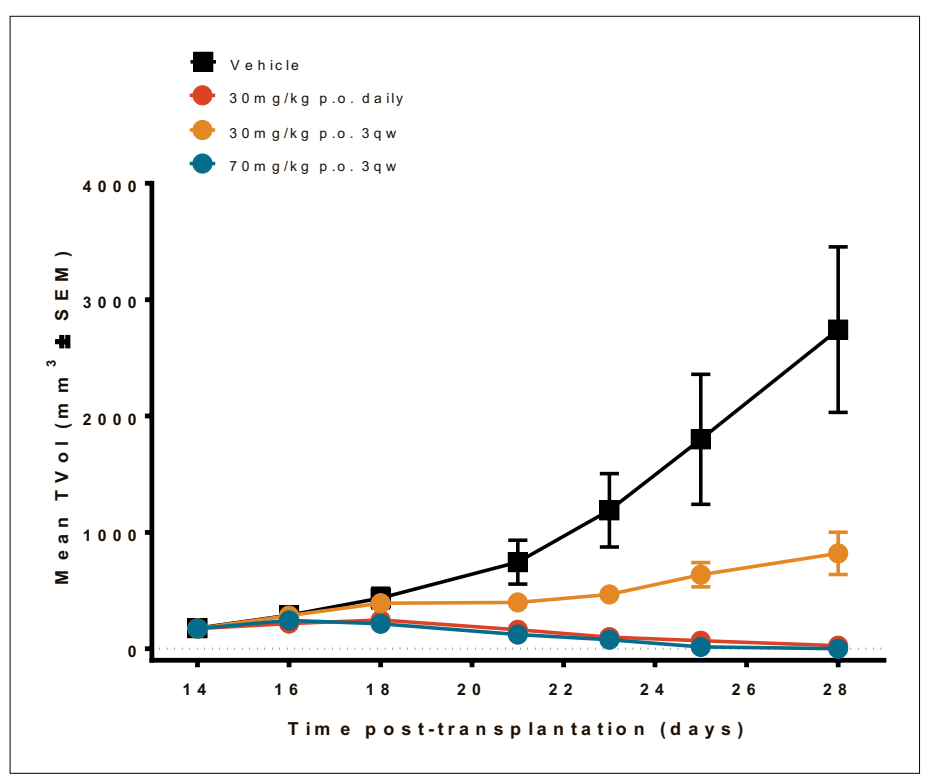

cept. Whereas the initial starting point had been identified by virtual screening that assumed to adopt a binding mode conforming to the 'central valine concept', the dihydroisoquinolinone compounds had been shown to adopt a very different binding mode. Furet et al. very recently published a detailed explanation for this, with a key aspect being that the dihydroisoquinolinone ring is not completely flat and the chlorophenyl group occupying the Trp-23 sub-pocket adopts a pseudo-axial conformation. ${ }^{[17]} A b$ initio calculations indicate that the pseudo-axial conformation is more stable than its pseudo-equatorial counterpart by $1.6 \mathrm{kcal} / \mathrm{mol}$. The pseudo-equato- 
rial conformation was necessary to fit the MDM2 cavity in our initial binding model (Fig. 2). Although a conformational energy penalty for binding in the above range is not prohibitive, as it can be overcome by either a few efficient van der Waals contacts or a productive hydrogen-bond interaction within the binding site, we reasoned that it would be advantageous to favor the putative MDM2 bound pseudo-equatorial conformation. For this we hypothesized that replacing the six-membered lactam ring of the dihydroisoquinolinone core by a five-membered lactam ring which, when fused to an aromatic ring, would be flat and project any substituent at the sp3 carbon position into an obligatory pseudoequatorial orientation. Examining this idea by interactive molecular modeling, we also realized that replacing the phenyl part of the dihydroisoquinolinone bicycle by, for example, an aromatic five-membered ring would provide two excellent exit vectors that could be exploited to fill the Phe-19 sub-pocket of MDM2. This is illustrated in Fig. 6 by a generic representation of such a fused 5-5 bicyclic system bearing substituents conforming to the Leu-26 and Trp-23 sub-pocket pharmacophores previously established, namely a meta-chlorophenyl ring for the former and a para-chlorophenyl ring for the latter. Before embarking in the synthesis of a prototype compound, we searched the Novartis compound collection for suitable compounds to test this hypothesis. Thus, the compound collection was searched using the broad substructure query as illustrated in the generic representation shown in Fig. 6. The search returned only one available compound retaining the critical chlorine atom in para-position of the phenyl ring intended to fill the Trp-23 sub-pocket. This was the pyrazolopyrrolidinone derivative 7 . To our delight, compound 7 inhibited the p53-MDM2 interaction with an $\mathrm{IC}_{50}$ value of $1.5 \mu \mathrm{M}$ in our biochemical TR-FRET assay despite being suboptimal, as the meta-chloro substituent on the phenyl ring targeting the Leu-26 sub-pocket as well as an optimal group filling the Phe-19 pocket were absent in the molecule. As a consequence we initiated a broader exploration to understand the potential of this new chemical series.

Our wide-ranging chemistry program started with the synthesis of a short series of analogs. Replacing the para-methyl group by a meta-chloro atom in the phenyl ring of 7 , assumed to bind in the Leu-26 sub-pocket, afforded a significant improvement of biochemical activity, consistent with our binding mode hypothesis (compound 8). An important feature of the Leu-26 subpocket pharmacophore identified in our previous series of inhibitors was the $\pi-\pi$ stacking interaction of the chlorophenyl ring with MDM2 residue His-96. ${ }^{[12]}$ For efficient stacking, the planes of the interacting aromatic rings need to be parallel. In the binding model of our pyrazolopyrrolidinone derivatives, this required the meta-chlorophenyl ring to be perpendicular to the plane of the lactam ring to which it is attached. Hence an ortho-substituent, such as a methyl group would be favorably preorganize the group to occupy the Leu-26 sub-pocket. Similarly, analogues of having larger hydrophobic substituents at position
3 of the pyrazole ring were designed to better fill the region of the MDM2 Phe-19 sub-pocket facing this position in the binding model. Again in support of the model, we established that an isopropyl group was optimal in this position. Although we now achieved potencies in the low double digit nanomolar range in the biochemical assay for these early hits, an opportunity remained to further increase the affinity of the pyrazolopyrrolidinone inhibitors for MDM2.

As can be appreciated from Fig. 6, the exit vector to access the Phe-19 sub-pocket was not exploited. This corresponds to the $\mathrm{N} 2$ position of the pyrazole ring of the inhibitors. We therefore designed various analogues bearing substituents at the $\mathrm{N} 2$ position to occupy the part of the Phe-19 subpocket extending along this vector in the binding model. The resulting compounds which are rigid would be preorganized for binding and the phenyl ring substituent would occupy a location in the model to allow small hydrophobic substituents to make additional favorable contacts with the MDM2 cavity. It turned out that the attachment of an ortho-methoxyphenyl group as shown in compound $\mathbf{9}$, produced a significant increase in potency. In fact, the active enantiomer of compound 9 displayed a spectacular $0.13 \mathrm{nM}$ potency in the TR-FRET assay. This sub-nanomolar activity in the biochemical assay translated well into a $\mathrm{GI}_{50}$ value of $90 \mathrm{nM}$ in the cellular SJSA-1 proliferation assay.

To understand the origin of the outstanding affinity of $\mathbf{9}$ for MDM2, we desired an X-ray co-crystal structure of it in<smiles>[R]C1CCC2=C(CC1)C(c1ccc(Cl)cc1)N(c1cccc(Cl)c1)C([R])C2</smiles><smiles>Cc1n[nH]c2c1C(c1ccc(Cl)cc1)N(c1cccc(Cl)c1)C2=O</smiles>

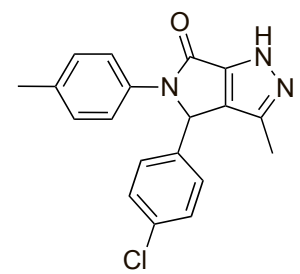

7

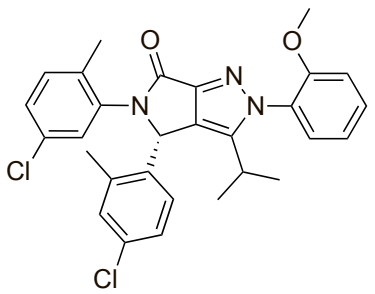

9

Fig. 6. New hypothesis to maintain the initial binding mode. The six-membered lactam ring of the isoquinolinone core is now replaced with a fivemembered lactam. Compound 7 represents the initial hit from the Novartis compound collection. Next to it, the binding model prediction of compound 7 bound to the cavity of MDM2. 
complex with MDM2. Based on previous experiences, we knew that increasing the solubility of the compound would improve the chance to obtain a co-crystal structure and consequently compound $\mathbf{1 0}$ was prepared. In 10, a basic dimethylaminoethanol group replaces the methyl in ortho position of the meta-chlorophenyl group of 9 and faces solvent in the binding model. We expected that such a solubilizing group extending towards the solvent would not disturb biochemical activity. This strategy was successful since $\mathbf{1 0}$ maintained the exceptional potency and we obtained a high resolution X-ray structure of it in complex with MDM2.

As shown in Fig. 7, we were delighted to see that in the crystal structure compound 10 adopts the anticipated 'central valine' binding mode. The pyrazolopyrrolidinone core of the compound made broad van der Waals contacts with the central MDM2 residue Val-93 while accepting a hydrogen-bond from the side-chain of His-96. Also in agreement with our binding mode hypothesis, the chlorophenyl moieties of the inhibitor occupy the Trp-23 and Leu19 sub-pockets, a $\pi$ - $\pi$ stacking interaction with His-96 being observed in the latter. In addition, the X-ray structure allowed us to rationalize why the ortho-methoxyphenyl moiety increased the potency. Within the Phe-19 sub-pocket, a favorable sulfur-aromatic interaction occurred between the phenyl ring and the sulfur atom of the side chain of residue M62.

The highly potent inhibitors 9 and $\mathbf{1 0}$ provided starting points for an extensive optimization program to find analogues suitable for in vivo studies and the detailed SAR and optimization work will be reported in due time elsewhere. This program has culminated in the discovery of NVP-HDM201 (Fig. 8), an imidazolopyrrolidinone analog, showing a very favorable in vitro and in vivo profile. ${ }^{[18,19]}$ The compound binds to human MDM2 in the picomolar range in the biochemical assay and shows anti-proliferative activity in the SJSA-1 cell line in the low nanomolar range. In our preclinical in vivo xenograft models, NVP-HDM201 is up to 10 -fold more potent compared to our NVP-CGM097. NVP-HDM201 possesses advantageous physicochemical and good drug-like properties which supported the selection of the compound for clinical development. NVP-HDM201 has now entered phase 1 clinical trials where it is evaluated as a single agent or in combinations in patients bearing tumors expressing wild-type p53.

Over the past several years, numerous other small molecule MDM2 inhibitors have also progressed into clinical trials and a compilation of known chemical matter has recently been published. ${ }^{[20]}$ Parallel to

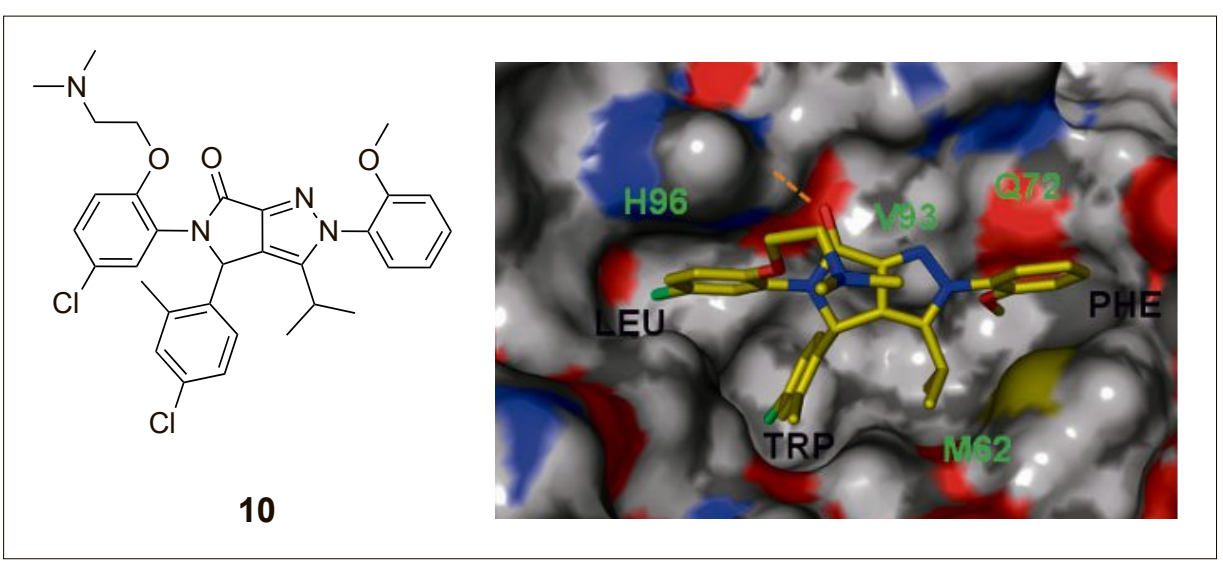

Fig. 7. Co-crystal structure of MDM2 in complex with compound 10 (PDB code: 5LN2). Compound 10 adopts the expected 'central valine' binding mode and the substituted chlorophenyl ring occupies the Leu-26 sub-pocket where it can interact via $\pi$ - $\pi$-stacking with His- 96 . The ortho-methoxyphenyl group occupying the Phe-19 sub-pocket sits in a small hydrophobic region in the back of the MDM2 cavity not accessible to solvent and as a result contributes to the potency increase of 10.

the p53-MDM2 PPI disruptors obtained by in silico approaches, starting points for drug discovery programs have also been found by screening proprietary compound collections. ${ }^{[21]}$ Using HTS, Roche scientists discovered imidazoline derivatives as the first non-peptidic inhibitors, structural biology (X-ray and NMR) guided optimization of which furnished the 2,4,5-triaryl derivative RG7112, whose phenyl groups mimic features of the Phe19-Trp23-Leu26 triad in p53, actually as the first clinical development candidate (Fig. 9). ${ }^{[22]}$ Using a different approach to lead-finding, Wang and coworkers (University of Michigan) applied structure-based design to identify a spiro-oxindole core structure which they elegantly elaborated, employing a 1,3-dipolar cycloaddition synthetic strategy, to mimic the same triad in $\mathrm{p} 53$, resulting in compounds that bind MDM2 with low nanomolar affinity.[23,24] In collaboration with industrial scientists (Ascenta Therapeutics and Sanofi), further optimization of these compounds for oral efficacy in mouse xenograft models and to finetuning their pharmacological properties, resulted in the identification of Sanofi's SAR405838 as a clinical candidate. ${ }^{[25]}$ By incorporating features from the spirooxindoles into their imidazoline-chemotype, the Roche scientists subsequently identified idasanutlin (often still referred to as RG7388), a more potent and selective follow-up compound to RG7112, that entered clinical development and currently is under evaluation in clinical phase III trials. ${ }^{[26]}$ Medicinal chemists at Amgen have similarly used rational structure-based design to identify and optimize a piperidine scaffold, leading to the discovery of the prototype piperidinone AM-8553 and culminating in AMG-232 that commenced clinical evaluation a few years ago. ${ }^{[27,28]}$

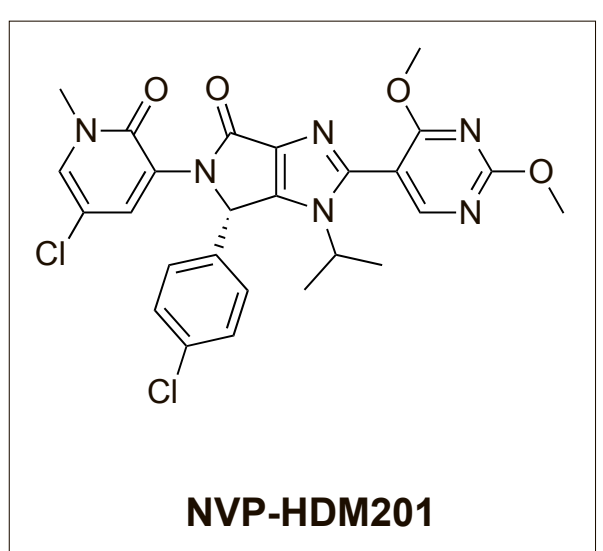

Fig. 8. Structure of NVP-HDM201, an imidazolopyrrolidinone analogue, is Novartis' second p53-MDM2 inhibitor in clinical development. The highly potent inhibitors $\mathbf{9}$ and $\mathbf{1 0}$ provided starting points for an extensive optimization discovery of NVP-HDM201.

And finally, scientists at Daiichi-Sankyo have discovered DS-3032b, as well an orally available MDM2 inhibitor which undergoes clinical development. [29]

The number of players in this area reflects the perceived potential of p53MDM2 inhibitors and successful medicinal chemistry endeavors have increased the number of agents selected for evaluation as treatments for a variety of solid tumors and hematological malignancies. Encouraging early clinical activity has been presented and full reports from studies with p53MDM2 inhibitors, as monotherapies and in combination are eagerly anticipated.

\section{Acknowledgement}

The author would like to acknowledge all Novartis colleagues who have being involved over so many years and with great dedication in the discovery of NVP-CGM097 and NVPHDM201. Also special thanks go to the numerprogram and these efforts culminated in the 


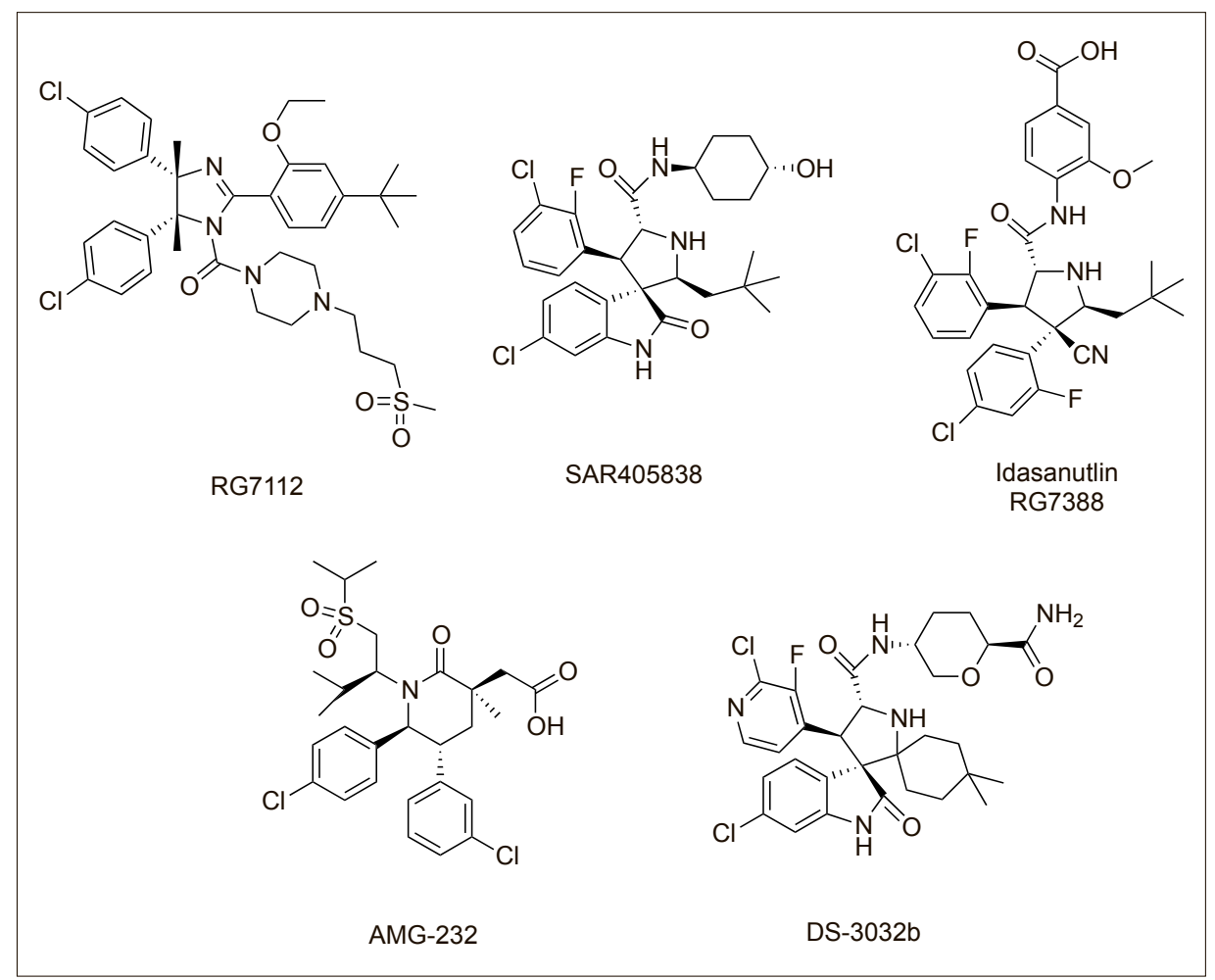

Fig. 9. Competitive landscape of published p53-MDM2 inhibitors evaluated in clinical trials.

ous associates for their technical assistance in preparing the compounds. I especially thank Paul Manley for his careful review of the manuscript and Pascal Furet for his support in generating the figures.

Received: July 27, 2017

[1] S. L. Harris, A. J. Levine, Oncogene 2005, 24, 2899.

[2] D. P. Lane, Nature 1992, 358, 15.

[3] D. Michael, M. Oren, Semin. Cancer Biol. 2003, 13, 49 .

[4] C. J. Kemp, L. A. Donehower, A. Bradley, A. Balmain, Cell 1993, 74, 813 .

[5] A. Feki, I. Irminger-Finger, Crit. Rev. Oncol. Hematol. 2004, 52, 103.

[6] J.-P. Kruse, W. Gu, Cell 2009, 137, 609.

[7] K. H. Khoo, C. S. Verma, D. P. Lane, Nature Rev. Drug Discov. 2014, 13, 217.

[8] W. Garland R. Benezra, J. Chaudhary, Ann. Rep. Med. Chem. 2013, 48, 227.

[9] P. H. Kussie, S. Gorina, V. Marechal, B. Elenbaas, J. Moreau, A. J. Levine, N. P. Pavletich, Science 1996, 274, 948.

[10] C. García-Echeverría, P. Chène, M. Blommers, P. Furet, J. Med. Chem. 2000, 43, 3205.

[11] P. Furet, P. Chene, A. De Pover, T. Valat, J. Hergovich Lisztwan, J. Kallen, K. Masuya, Bioorg. Med. Chem. Lett. 2012, 22, 3498.

[12] A. Vaupel, G. Bold, A. De Pover, T. StachyraValat, J. Hergovich Lisztwan, J. Kallen, K. Masuya, P. Furet, Bioorg. Med. Chem. Lett. 2014, 24, 2110.

[13] E. Jacoby, A. Boettcher, L. Mayr, N. Brown, J. Jenkins, J. Kallen, C. Engeloch, U. Schopfer, P. Furet, K. Masuya, Methods Mol. Biol. 2009 , $575,173$.

[14] F. Gessier, J. Kallen, E. Jacoby, P. Chène, T. Stachyra-Valat, S. Ruetz, S. Jeay, P. Holzer, K. Masuya, P. Furet, Bioorg. Med. Chem. Lett., 2015, 25, 3621.
[15] P. Holzer, K. Masuya, P. Furet, J. Kallen, T. Valat-Stachyra, S. Ferretti, J. Berghausen, M. Bouisset-Leonard, N. Buschmann, C. PissotSoldermann, C. Rynn, S. Ruetz, S. Stutz, P. Chène, S. Jeay, F. Gessier, J. Med. Chem. 2015, 58, 6348.

[16] S. Jeay, S. Gaulis, S. Ferretti, H. Bitter, M. Ito, T. Valat, M. Murakami, S. Ruetz, D. A. Guthy, C. Rynn, M. R. Jensen, M. Wiesmann, J. Kallen, P. Furet, F. Gessier, P. Holzer, K. Masuya, J. Würthner, E. Halilovic, F. Hofmann, W. R. Sellers, D. Graus Porta, eLife 2015, DOI: 10.7554/eLife.06498

[17] P. Furet, K. Masuya, J. Kallen, T. StachyraValat, S. Ruetz, V. Guagnano, P. Holzer, R. Mah, S. Stutz, A. Vaupel, P. Chène, S. Jeay, A. Schlapbach, Bioorg. Med. Chem. Lett. 2016, 26, 4837.

[18] P. Holzer, P. Chene, S. Ferretti, P. Furet, T. Gabriel, B. Gruenenfelder, V. Guagnano, F. Hofmann, J. Kallen, R. Mah, K. Masuya, R. Ramos, S. Ruetz, C. Rynn, A. Schlapbach, T. Stachyra-Valat, S. Stutz, A. Vaupel, S. Jeay, Abstracts of Papers, 252nd ACS National Meeting \& Exposition, Philadelphia, PA, United States, August 21-25, 2016, abstract no. MEDI-273.

[19] P. Holzer, P. Chene, S. Ferretti, P. Furet, T. Gabriel, B. Gruenenfelder, V. Guagnano, F. Hofmann, J. Kallen, R. Mah, K. Masuya, R. Ramos, S. Ruetz, C. Rynn, A. Schlapbach, T. Stachyra-Valat, S. Stutz, A. Vaupel, S. Jeay, AACR Annual Meeting 2016, New Orleans, LA, United States, April 16-20, 2016, abstract no. 4855.

[20] C. Neochoritis, N. Estrada-Ortiz, K. Khoury, A. Dömling, Annu. Rep. Med. Chem. 2014, 49, 167.

[21] L. T. Vassilev, B. T. Vu, B. Graves, D. Carvajal, F. Podlaski, Z. Filipovic, N. Kong, U. Kammlott, C. Lukacs, C. Klein, N. Fotouhi, E. A. Liu, Science 2004, 303, 844.

[22] Q. Ding, Z. Zhang, J. Liu, N. Jiang, J. Zhang, T. M. Ross, X. Chu, D. Bartkovitz, F. Podlaski, C. Janson, C. Tovar, Z. M. Filipovic, B. Higgins,
K. Glenn, K. Packman, L. Vassilev, B. Graves, J. Med. Chem. 2013, 56, 5979.

[23] S. Yu, D. Qin, S. Shangary, J. Chen, G. Wang, K. Ding, D. McEachern, S. Qiu, Z. NikolovskaColeska, R. Miller, S. Kang, D. Yang, S. Wang, J. Med. Chem. 2009, 52, 7970.

[24] S. Wang, Y. Zhao, D. Bernard, A. Aguilar, S. Kumar, Top. Med. Chem. 2012, 8, 57 .

[25] S. Wang, W. Sun, Y. Zhao, D. McEachern, I. Meaux, C. Barrière, J. A. Stuckey, J. L. Meagher, L. Bai, L. Liu, C. G. Hoffman-Luca, J. Lu, S. Shangary, S. Yu, D. Bernard, A. Aguilar, O. Dos-Santos, L. Besret, S. Guerif, P. Pannie, D. Gorge-Bernat, L. Debussche, Cancer Res. 2014, 74, 5855.

[26] Q. Ding, Z. Zhang, J. Liu, N. Jiang, J. Zhang, T M. Ross, X. Chu, D. Bartkovitz, F. Podlaski, C. Janson, C. Tovar, Z. M. Filipovic, B. Higgins, K. Glenn, K. Packman, L. Vassilev, B. Graves, J. Med. Chem. 2013, 56, 5979.

[27] Y. Rew, D. Sun, F. Gonzalez-Lopez De Turiso, M. D. Bartberger, H. P. Beck, J. Canon, A. Chen, D. Chow, J. Deignan, B. M. Fox, D Gustin, X. Huang, M. Jiang, X. Jiao, L. Jin, F. Kayser, D. J. Kopecky, Y. Li, M. C. Lo, A. M. Long, K. Michelsen, J. D. Oliner, T. Osgood, M. Ragains, A. Y. Saiki, S. Schneider, M. Toteva, P. Yakowec, X. Yan, Q. Ye, D. Yu, X. Zhao, J. Zhou, J. C. Medina, S. H. Olson, J. Med. Chem. 2012, 55, 4936.

[28] D. Sun, Z. Li, Y. Rew, M. Gribble, M. D. Bartberger, H. P. Beck, J. Canon, A. Chen, X. Chen, D. Chow, J. Deignan, J. Duquette, J. Eksterowicz, B. Fisher, B. M. Fox, J. Fu, A. Z. Gonzalez, F. Gonzalez-Lopez De Turiso, J. B. Houze, X. Huang, M. Jiang, L. Jin, F. Kayser, J. Liu, M. C. Lo, A. M. Long, B. Lucas, L. R. McGee, J. McIntosh, J. Mihalic, J. D. Oliner, T. Osgood, M. L. Peterson, P. Roveto, A. Y. Saiki, P. Shaffer, M. Toteva, Y. Wang, Y. C. Wang, S. Wortman, P. Yakowec, X. Yan, Q. Ye, D. Yu, M. Yu, X. Zhao, J. Zhou, J. Zhu, S. H. Olson, J. C. Medina, J. Med. Chem. 2014, 57, 1454.

[29] C. D. DiNardo, J. Rosenthal, M. Andreeff, O. Zernovak, P. Kumar, R. Gajee, S. Chen, M. Rosen, S. Song, J. Kochan, T. Limsakun, R. Olin, 'Phase 1 Dose Escalation Study of MDM2 Inhibitor DS-3032b in Patients with Hematological Malignancies - Preliminary Results', American Society of Hematology, 58th Annual Meeting and Exposition, San Diego, CA, United States, December 3-6, 2016, no. 593. 